\title{
Multiple solutions for a fourth-order nonlinear elliptic problem which is superlinear at $+\infty$ and linear at $-\infty$
}

Ruichang Pei ${ }^{1,2^{*}}$ and Jihui Zhang ${ }^{2}$

"Correspondence: prc211@163.com ${ }^{1}$ School of Mathematics and Statistics, Tianshui Normal University, Tianshui, 741001, P.R. China

${ }^{2}$ School of Mathematics and Computer Sciences, Nanjing Normal University, Nanjing, 210097, P.R. China

\begin{abstract}
We consider a semilinear fourth-order elliptic equation with a right-hand side nonlinearity which exhibits an asymmetric growth at $+\infty$ and at $-\infty$. Namely, it is linear at $-\infty$ and superlinear at $+\infty$. Combining variational methods with Morse theory, we show that the problem has at least two nontrivial solutions, one of which is negative.
\end{abstract}

Keywords: fourth-order elliptic boundary value problems; multiple solutions; critical groups; Morse theory

\section{Introduction}

Consider the following Navier boundary value problem:

$$
\begin{cases}\triangle^{2} u(x)+c \Delta u=f(x, u) & \text { in } \Omega, \\ u=\Delta u=0 & \text { on } \partial \Omega\end{cases}
$$

where $\triangle^{2}$ is the biharmonic operator, and $\Omega$ is a bounded smooth domain in $\mathbb{R}^{N}(N>4)$, and $c<\lambda_{1}^{*}$ the first eigenvalue of $-\triangle$ in $H_{0}^{1}(\Omega)$.

The conditions imposed on $f(x, t)$ are as follows:

$\left(\mathrm{H}_{1}\right) f \in C^{1}(\bar{\Omega} \times \mathbb{R}, \mathbb{R}), f(x, 0)=0$ for all $x \in \Omega$ and $f(x, t)<0$ for all $t<0$ and all $x \in \Omega$;

$\left(\mathrm{H}_{2}\right)$ there exist $r \in\left(2, p^{*}\right)$ and $A, B>0$ such that $\left|f_{t}^{\prime}(x, t)\right| \leq A+B|t|^{r-2}$ for all $x \in \Omega$, and $t \in \mathbb{R}$, where $p^{*}=\frac{2 N}{N-4}$, if $N>4$;

$\left(\mathrm{H}_{3}\right) \lim _{t \rightarrow-\infty} \frac{f(x, t)}{t}=l$ uniformly for $x \in \Omega$, where $l$ is a nonnegative constant;

$\left(\mathrm{H}_{4}\right)$ there exist $\beta, \xi \in \mathbb{R}$ such that for $F(x, t)=\int_{0}^{t} f(x, s) d s$, we have

$$
\begin{aligned}
& \lim _{t \rightarrow-\infty} \sup (2 F(x, t)-f(x, t) t) \leq \beta \quad \text { uniformly for all } x \in \Omega, \\
& \lim _{t \rightarrow+\infty} \frac{F(x, t)}{t^{2}}=+\infty \quad \text { uniformly for all } x \in \Omega
\end{aligned}
$$

and

$$
\lim _{t \rightarrow+\infty} \inf (f(x, t) t-2 F(x, t)) \geq \xi \quad \text { uniformly for all } x \in \Omega
$$

O2014 Pei and Zhang; licensee Springer. This is an Open Access article distributed under the terms of the Creative Commons Attribution License (http://creativecommons.org/licenses/by/2.0), which permits unrestricted use, distribution, and reproduction in any medium, provided the original work is properly cited. 
$\left(\mathrm{H}_{5}\right)$ there exist $\vartheta_{1}, \vartheta_{2} \in L^{\infty}(\Omega)_{+}$and an integer $k \geq 2$ such that

$$
\lambda_{k} \leq \vartheta_{1}(x) \leq \vartheta_{2}(x) \leq \lambda_{k+1} \quad \text { for all } x \in \Omega
$$

the first and the last inequality are strict on sets (not necessary the same) of positive measure, and

$$
\vartheta_{1}(x) \leq f_{t}^{\prime}(x, 0)=\lim _{t \rightarrow 0} \frac{f(x, t)}{t} \leq \vartheta_{2}(x) \quad \text { uniformly for all } x \in \Omega
$$

In view of the conditions $\left(\mathrm{H}_{3}\right)$ and equation $(3)$ in $\left(\mathrm{H}_{4}\right)$, it is clear that for all $x \in \Omega, f(x, t)$ is linear at $-\infty$ and superlinear at $+\infty$. Clearly, $u=0$ is a trivial solution of problem (1). It follows from $\left(\mathrm{H}_{1}\right)$ and $\left(\mathrm{H}_{2}\right)$ that the functional

$$
I(u)=\frac{1}{2} \int_{\Omega}\left(|\Delta u|^{2}-c|\nabla u|^{2}\right) d x-\int_{\Omega} F(x, u) d x
$$

is $C^{2}$ on the space $H_{0}^{1}(\Omega) \cap H^{2}(\Omega)$ with the norm

$$
\|u\|:=\left(\int_{\Omega}\left(|\Delta u|^{2}-c|\nabla u|^{2}\right) d x\right)^{\frac{1}{2}}
$$

where $F(x, t)=\int_{0}^{t} f(x, s) d s$. Under the condition $\left(\mathrm{H}_{2}\right)$, the critical points of $I$ are solutions of problem (1). Let $0<\lambda_{1}<\lambda_{2}<\cdots<\lambda_{k}<\cdots$ be the eigenvalues of $\left(\triangle^{2}+c \triangle, H^{2}(\Omega) \cap\right.$ $\left.H_{0}^{1}(\Omega)\right)$ and $\phi_{1}(x)>0$ be the eigenfunction corresponding to $\lambda_{1}$. In fact, $\lambda_{1}=\lambda_{1}^{*}\left(\lambda_{1}^{*}-c\right)$. Let $E_{\lambda_{k}}$ denote the eigenspace associated with $\lambda_{k}$. Throughout this article, we denoted by $|\cdot|_{p}$ the $L^{p}(\Omega)$ norm and $E=H^{2}(\Omega) \cap H_{0}^{1}(\Omega)$. The aim of this paper is to prove a multiplicity theorem for problem (1) when the nonlinearity term $f(x, t)$ exhibits an asymmetric behavior as $t \in \mathbb{R}$ approaches $+\infty$ and $-\infty$. In the past, some authors studied the following elliptic problem:

$$
-\triangle u=f(x, u), \quad u \in H_{0}^{1}(\Omega)
$$

with asymmetric nonlinearities by using the Fučík spectrum of the operator $\left(-\triangle, H_{0}^{1}(\Omega)\right.$ ). This approach requires that $f(x, t)$ exhibits linear growth at both $+\infty$ and $-\infty$ and that the limits $\lim _{t \rightarrow \pm \infty} \frac{f(x, t)}{t}$ exist and belong to $\mathbb{R}$. See the works of Các [1], Dancer and Zhang [2], Magalhães [3], de Paiva [4], Schechter [5] and the references therein. Equations with nonlinearities which are superlinear in one direction and linear in the other were investigated by Arcoya and Villegas [6] and Perera [7]. They let the nonlinearity $f(x, t)$ be line at $-\infty$ and satisfy the Ambrosetti-Rabinowitz condition at $+\infty$. Particularly, it is worth noticing paper [8]. The authors relax several of the above restrictions on the nonlinearity $f(x, t)$. Their nonlinearity is only measurable in $x \in \Omega$. The limit as $t \rightarrow-\infty$ of $\frac{f(x, t)}{t}$ need not exist and the growth at $-\infty$ can be linear or sublinear. Furthermore, their nonlinearity $f(x, t)$ does not satisfy the famous AR-condition. They use the truncated skill of first order weak derivative to verify the (PS) condition and obtain multiple solutions for problem (1) by combining variational methods and Morse theory.

To the authors' knowledge, there seem to be few results on problem (1) when $f(x, t)$ is asymmetric nonlinearity at positive infinity and at negative infinity. However, the 
method in [8] cannot be applied directly to the biharmonic problems. For example, for the Laplacian problem, $u \in H_{0}^{1}(\Omega)$ implies $|u|, u_{+}, u_{-} \in H_{0}^{1}(\Omega)$, where $u_{+}=\max (u, 0)$, $u_{-}=\max (-u, 0)$. We can use $u_{+}$or $u_{-}$as a test function, which is helpful in proving a solution nonnegative. While for the biharmonic problems, this trick fails completely since $u \in H_{0}^{2}(\Omega)$ does not imply $u_{+}, u_{-} \in H_{0}^{2}(\Omega)$ (see [9, Remark 2.1.10] and [10, 11]). As far as this point is concerned, we will make use of the new methods to overcome it.

This fourth-order semilinear elliptic problem can be considered as an analogue of a class of second-order problems which have been studied by many authors. In [12], there was a survey of results obtained in this direction. In [13], Micheletti and Pistoia showed that $\left(P_{1}\right)$ admits at least two solutions by a variation of linking if $f(x, u)$ is sublinear. Chipot [14] proved that the problem $\left(P_{1}\right)$ has at least three solutions by a variational reduction method and a degree argument. In [15], Zhang and Li showed that $\left(P_{1}\right)$ admits at least two nontrivial solutions by Morse theory and local linking if $f(x, u)$ is superlinear and subcritical on $u$.

In this article, under the guidance of [8], we consider multiple solutions of problem (1) with the asymmetric nonlinearity by using variational methods and Morse theory.

\section{Main result and auxiliary lemmas}

Let us now state the main result.

Theorem 2.1 Assume conditions $\left(\mathrm{H}_{1}\right)-\left(\mathrm{H}_{5}\right)$ hold. If $l<\lambda_{1}$, then problem (1) has at least two nontrivial solutions.

Lemma 2.2 Under the assumptions of Theorem 2.1, then I satisfies the (PS) condition.

Proof Let $\left\{u_{n}\right\} \subset E$ be a sequence such that for every $n \in N$,

$$
\begin{aligned}
& \left|\frac{1}{2} \int_{\Omega}\left(\left|\Delta u_{n}\right|^{2}-c\left|\nabla u_{n}\right|^{2}\right) d x-\int_{\Omega} F\left(x, u_{n}\right) d x\right| \leq c, \\
& \left|\int_{\Omega}\left(\Delta u_{n} \Delta v-c \nabla u_{n} \nabla v\right) d x-\int_{\Omega} f\left(x, u_{n}\right) v d x\right| \leq \varepsilon_{n}\|v\|, \quad v \in E,
\end{aligned}
$$

where $c>0$ is a positive constant and $\left\{\varepsilon_{n}\right\} \subset \mathbb{R}^{+}$is a sequence which converges to zero. By a standard argument, in order to prove that $\left\{u_{n}\right\}$ has a convergence subsequence, we have to show that it is a bounded sequence. To do this, we argue by contradiction assuming that for a subsequence, denoted by $\left\{u_{n}\right\}$, we have

$$
\left\|u_{n}\right\| \rightarrow+\infty \quad \text { as } n \rightarrow \infty
$$

Without loss of generality we can assume $\left\|u_{n}\right\|>1$ for all $n \in \mathbb{N}$ and define $z_{n}=\frac{u_{n}}{\left\|u_{n}\right\|}$. Obviously, $\left\|z_{n}\right\|=1 \forall n \in \mathbb{N}$ and then it is possible to extract a subsequence (denoted also by $\left.\left\{z_{n}\right\}\right)$ such that

$$
\begin{aligned}
& z_{n} \rightarrow z_{0} \quad \text { in } E, \\
& z_{n} \rightarrow z_{0} \quad \text { in } L^{2}(\Omega), \\
& z_{n}(x) \rightarrow z(x), \quad \text { a.e. } x \in \Omega, \\
& \left|z_{n}(x)\right| \leq q(x), \quad \text { a.e. } x \in \Omega,
\end{aligned}
$$


where $z_{0} \in E$ and $q \in L^{2}(\Omega)$. Dividing both sides of inequality (10) by $\left\|u_{n}\right\|$, we obtain

$$
\left|\int_{\Omega}\left(\triangle z_{n} \Delta v-c \nabla z_{n} \nabla v\right) d x-\int_{\Omega} \frac{f\left(x, u_{n}\right)}{\left\|u_{n}\right\|} \nu d x\right| \leq \frac{\varepsilon_{n}}{\left\|u_{n}\right\|}\|v\| \quad \text { for all } v \in E .
$$

Passing to the limit we deduce from equation (11) that

$$
\lim _{n \rightarrow \infty} \int_{\Omega} \frac{f\left(x, u_{n}\right)}{\left\|u_{n}\right\|} v d x=\int_{\Omega}\left(\triangle z_{0} \Delta v-c \nabla z_{0} \nabla v\right) d x
$$

for all $v \in E$.

Now we claim that $z_{0}(x) \leq 0$ a.e. $x \in \Omega$. To verify this, let us observe that by choosing $\nu=z_{0}$ in equation (15) we have

$$
\lim _{n \rightarrow \infty} \int_{\Omega^{+}} \frac{f\left(x, u_{n}\right)}{\left\|u_{n}\right\|} z_{0} d x<+\infty
$$

where $\Omega^{+}=\left\{x \in \Omega \mid z_{0}(x)>0\right\}$. But, on the other hand, from $\left(\mathrm{H}_{3}\right)$ and equation (3) in $\left(\mathrm{H}_{4}\right)$, we have

$$
\frac{f\left(x, u_{n}(x)\right)}{\left\|u_{n}\right\|} z_{0}(x) \geq \frac{l u_{n}(x)-K_{1}}{\left\|u_{n}\right\|} z_{0}(x) \geq\left(-l q(x)-K_{1}\right) z_{0}(x), \quad \text { a.e. } x \in \Omega
$$

for some positive constant $K_{1}>0$. Moreover, using $\lim _{n \rightarrow \infty} u_{n}(x)=+\infty$ a.e. $x \in \Omega^{+}$, equation (13) and the superlinearity of $f$, we also deduce

$$
\lim _{n \rightarrow \infty} \frac{f\left(x, u_{n}(x)\right)}{\left\|u_{n}\right\|} z_{0}(x)=\lim _{n \rightarrow \infty} \frac{f\left(x, u_{n}(x)\right)}{u_{n}} z_{n}(x) z_{0}(x)=+\infty, \quad \text { a.e. } x \in \Omega^{+} .
$$

Therefore, if $\left|\Omega^{+}\right|>0$ we will obtain by Fatou's lemma that

$$
\lim _{n \rightarrow \infty} \int_{\Omega^{+}} \frac{f\left(x, u_{n}(x)\right)}{\left\|u_{n}\right\|} z_{0}(x) d x=+\infty
$$

which contradicts inequality (16). Thus $\left|\Omega^{+}\right|=0$ and the claim is proved.

Clearly, $z_{0}(x) \not \equiv 0$, by $\left(\mathrm{H}_{3}\right)$, there exists $C>0$ such that $\frac{\left|f\left(x, u_{n}\right)\right|}{\left|u_{n}\right|} \leq C$ for a.e. $x \in \Omega$. By using Lebesgue dominated convergence theorem in equation (15), we have

$$
\int_{\Omega}\left(\Delta z_{0} \Delta v-c \nabla z_{0} \nabla v\right) d x-\int_{\Omega} l z_{0} v d x=0
$$

for all $v \in E$. This contradicts $l<\lambda_{1}$.

Lemma 2.3 Let $E=V \oplus W$, where $V=E_{\lambda_{1}} \oplus E_{\lambda_{2}} \oplus \cdots \oplus E_{\lambda_{k}}$. If $k \geq 0$ is an integer, $\vartheta \in$ $L^{\infty}(\Omega)_{+}, \vartheta(x) \leq \lambda_{k+1}$ a.e. on $\Omega$ and the inequality is strict on a set of positive measure, then there exists $\gamma>0$ such that

$$
\|u\|^{2}-\int_{\Omega} \vartheta u^{2} d x \geq \gamma\|u\|^{2}
$$

for all $u \in W$. 
Proof We claim that there exists a constant $\vartheta_{0}<1$ such that

$$
\int_{\Omega} \vartheta(x) u^{2} d x \leq \vartheta_{0}\|u\|^{2}
$$

for all $u \in W$. In fact, if not, there exists a sequence $\left\{u_{n}\right\}$ such that

$$
\int_{\Omega} \vartheta(x)\left|u_{n}\right|^{2} d x>\left(1-\frac{1}{n}\right)\left\|u_{n}\right\|^{2}
$$

for all $n \in N$, which implies $u_{n} \neq 0$ for all $n$. By the homogeneity of the above inequality, we may assume that $\left\|u_{n}\right\|=1$ and

$$
\int_{\Omega} \vartheta(x)\left|u_{n}\right|^{2} d x>1-\frac{1}{n}
$$

for all $n$. It follows from the weak compactness of the unit ball of $W$ that there exists a subsequence, say $\left\{u_{n}\right\}$, such that $u_{n}$ weakly converges to $u$ in $W$. Now Sobolev's embedding theorem suggests that $\left\{u_{n}\right\}$ converges to $u$ in $L^{2}(\Omega)$. From inequality (19) we obtain

$$
\int_{\Omega} \vartheta(x)|u|^{2} d x \geq 1
$$

Moreover one has

$$
1 \geq\|u\|^{2} \geq \lambda_{k+1}|u|_{2}^{2} \geq \int_{\Omega} \vartheta(x)|u|^{2} d x \geq 1
$$

Hence we have

$$
\|u\|^{2}=\lambda_{k+1}|u|_{2}^{2}
$$

and

$$
\int_{\Omega}\left(\lambda_{k+1}-\vartheta(x)\right) u^{2} d x=0
$$

which implies that $u \in E_{\lambda_{k+1}} \backslash\{0\}$ and $u=0$ on a positive measure subset. It contradicts the unique continuation property of the eigenfunction.

\section{Computation of the critical groups}

It is well known that critical groups and Morse theory are the main tools in solving elliptic partial differential equation. Let us recall some results which will be used later. We refer the readers to the book [16] for more information on Morse theory.

Let $H$ be a Hilbert space and $I \in C^{1}(H, \mathbb{R})$ be a functional satisfying the (PS) condition or $(C)$ condition, and $H_{q}(X, Y)$ be the $q$ th singular relative homology group with integer coefficients. Let $u_{0}$ be an isolated critical point of $I$ with $I\left(u_{0}\right)=c, c \in \mathbb{R}$, and $U$ be a neighborhood of $u_{0}$. The group

$$
C_{q}\left(I, u_{0}\right):=H_{q}\left(I^{c} \cap U, I^{c} \cap U \backslash\left\{u_{0}\right\}\right), \quad q \in Z
$$

is said to be the $q$ th critical group of $I$ at $u_{0}$, where $I^{c}=\{u \in H: I(u) \leq c\}$. 
Let $K:=\left\{u \in H: I^{\prime}(u)=0\right\}$ be the set of critical points of $I$ and $a<\inf I(K)$, the critical groups of $I$ at infinity are formally defined by [17]

$$
C_{q}(I, \infty):=H_{q}\left(H, I^{a}\right), \quad q \in Z
$$

From the deformation theorem, we see that the above definition is independent of the particular choice of $c<\inf I(K)$. If $c<\inf I(K)$ then

$$
C_{q}(I, \infty):=H_{q}\left(H, \dot{I}^{a}\right), \quad q \in Z
$$

For the convenience of our proof, we first recall two interesting results and prove two important propositions.

Proposition 3.1 [18] Under $\left(\mathrm{H}_{2}\right)$, if $u \in E:=H^{2}(\Omega) \cap H_{0}^{1}(\Omega)$ is an isolated critical point of $I$, then $C_{*}(I, u) \cong C_{*}\left(\left.I\right|_{C_{0}^{3}(\Omega)}, u\right)$.

Proposition 3.2 [19] If $D_{1} \subset D \subset E_{0} \subset E_{1} \subset X$ and for some integer $k \geq 0$ we have $H_{k}(E, D) \neq 0$ and $H_{k}\left(E_{1}, D_{1}\right)=0$, then either $H_{k+1}\left(E_{1}, E\right) \neq 0$ or $H_{k-1}\left(D, D_{1}\right) \neq 0$.

Proposition 3.3 If the assumptions of Theorem 2.1 hold, then

$$
C_{k}(I, \infty)=0 \quad \text { for all integers } k \geq 0 \text {. }
$$

Proof Under the guidance of [8] and [18], we begin to prove this result. Let $I_{1}=\left.I\right|_{C_{0}^{3}(\bar{\Omega})}$. Indeed, it follows from above Proposition 3.1 that $I$ and $I_{1}$ have same critical set. Since $C_{0}^{3}(\bar{\Omega})$ is dense in $E$, invoking Proposition 16 of Palais [20], we have

$$
H_{k}\left(E, \dot{I}^{a}\right)=H_{k}\left(C_{0}^{3}(\bar{\Omega}), \dot{I}_{1}^{a}\right) \quad \text { for all } a \in R \text { and all integers } k \geq 0 \text {. }
$$

From equations (20) and (21), we see that in order to prove the proposition, it suffices to show that

$$
H_{k}\left(C_{0}^{3}(\bar{\Omega}), I_{1}^{a}\right)=0 \quad \text { for all } a<0 \text { with }|a| \text { large and all integers } k \geq 0 \text {. }
$$

In order to prove equation (22), we proceed as follows. We define the sets

$$
\partial B_{1}^{c}=\left\{u \in C_{0}^{3}(\bar{\Omega}):\|u\|_{C_{0}^{3}(\bar{\Omega})}=1\right\}
$$

and

$$
\partial B_{1,+}^{c}=\left\{u \in \partial B_{1}^{c}: u(x)>0 \text { for some } x \in \Omega\right\} .
$$

Consider the map $h_{+}:[0,1] \times \partial B_{1,+}^{c} \rightarrow \partial B_{1,+}^{c}$ defined by

$$
h_{+}(t, u)=\frac{(1-t) u+t \phi_{1}}{\left\|(1-t) u+t \phi_{1}\right\|_{C_{0}^{3}(\bar{\Omega})}} \quad \text { for all }(t, u) \in[0,1] \times \partial B_{1,+}^{c} .
$$

Clearly, $h_{+}$is a continuous homotopy and $h(1, u)=\phi_{1}$ for all $x \in \partial B_{1,+}^{c}$. Therefore, $\partial B_{1,+}^{c}$ is contractible in itself. 
By equation (3) in $\left(\mathrm{H}_{4}\right)$, given any $\gamma>0$, we can find $C=C(\gamma)>0$ such that

$$
F(x, t) \geq \frac{\gamma}{2} t^{2} \quad \text { for all } x \in \Omega \text { and all } t \geq C .
$$

Similarly, from condition $\left(\mathrm{H}_{3}\right)$, and by choosing $\mathrm{C}>0$ even bigger if necessary, we observe that there is a number $\gamma_{0}>0$ such that

$$
F(x, t) \geq-\frac{\gamma_{0}}{2} t^{2} \quad \text { for all } x \in \Omega \text { and all } t \leq-C(\gamma) .
$$

Moreover, by condition $\left(\mathrm{H}_{2}\right)$, we have

$$
|F(x, t)| \leq C_{3} \quad \text { for all } x \in \Omega \text { and all }|t| \leq C(\gamma)
$$

for some $C_{3}>0$.

Let $u \in \partial B_{1,+}^{c}$. By inequalities (23), (24), and (25), for all $t>0$ we have

$$
\begin{aligned}
I(t u) & =\frac{t^{2}}{2}\|u\|^{2}-\int_{\Omega} F(x, t u) d x \\
& =\frac{t^{2}}{2}\|u\|^{2}-\int_{t u \geq C} F(x, t u) d x-\int_{t u \leq-C} F(x, t u) d x-\int_{|t u| \leq C} F(x, t u) d x \\
& \leq \frac{t^{2}}{2}\left[\left(1+\frac{\gamma_{0}}{\lambda_{1}}\right)\|u\|^{2}-\gamma \int_{t u \geq C} u^{2} d x\right]+C_{3}|\Omega| .
\end{aligned}
$$

Recalling that $\gamma>0$ is arbitrary, from (26), we have

$$
I(t u) \rightarrow-\infty \quad \text { as } t \rightarrow-\infty .
$$

Using formula (4) in condition $\left(\mathrm{H}_{4}\right)$, we see that there exist constants $\xi_{0}$ and $M>0$ such that

$$
f(x, t) t-2 F(x, t) \geq \xi_{0} \quad \text { for all } x \in \Omega \text { and all } t \geq M
$$

By $\left(\mathrm{H}_{2}\right)$ and formula (2) in condition $\left(\mathrm{H}_{4}\right)$, we have

$$
2 F(x, t)-f(x, t) t \leq C \quad \text { for all } x \in \Omega \text { and all } t<M
$$

for some $C>0$. By inequalities (28) and (29), for any $u \in E$ we have

$$
\int_{\Omega}(2 F(x, t)-f(x, t) t) d x \leq C,
$$

where $C$ is a positive constant. Let $i: C_{0}^{3}(\bar{\Omega}) \rightarrow E$ be the continuous embedding map. Let $\langle\cdot, \cdot\rangle_{0}$ denote the duality brackets for the pair $\left(C_{0}^{3}(\bar{\Omega})^{*}, C_{0}^{3}(\bar{\Omega})\right)$. We let $I_{2}=I \circ i$, and so

$$
\begin{aligned}
& I_{2}^{\prime}(u)=i^{*} I^{\prime}(i(u)) \quad \text { for all } u \in C_{0}^{3}(\bar{\Omega}), \\
& \frac{d}{d t} I_{2}(t u)=\left\langle I_{2}^{\prime}(t u), u\right\rangle_{0}=t\|u\|^{2}-\int_{\Omega} f(x, t u) u d x \leq \frac{1}{t}\left(2 I(t u)+C^{*}\right) .
\end{aligned}
$$


Then, from equation (27), we obtain

$$
\frac{d}{d t} I_{2}(t u)<0 \text { for all } t>0 \text { large such that } I(t u)<-\frac{C^{*}}{2}
$$

From conditions $\left(\mathrm{H}_{2}\right)$ and $\left(\mathrm{H}_{3}\right)$, we see that given $\epsilon>0$, we can find $M>0$ such that

$$
F(x, t) \leq \frac{1}{2}(l+\epsilon) t^{2}+M \quad \text { for all } x \in \Omega \text { and all } t \leq 0 .
$$

Using inequality (33), we have

$$
\begin{aligned}
I(u) & \geq \frac{1}{2}\left(\|u\|^{2}-l|u|_{2}^{2}-\epsilon|u|_{2}^{2}\right)-M \\
& \geq C\|u\|^{2}-M
\end{aligned}
$$

for $u \in-C_{+}$, where $C_{+}$is defined as

$$
\left\{u \in C_{0}^{3}(\Omega): u(x) \geq 0 \text { for all } x \in \Omega\right\}
$$

and $C>0$ is a positive constant. So $\left.I\right|_{-C^{+}}$is coercive, thus we find $C^{* *}>0$ such that $\left.I\right|_{-C^{+}} \geq$ $-C^{* *}$. We pick

$$
a<\min \left\{-\frac{C^{*}}{2},-C^{* *}, \inf _{\partial B_{1}^{c}} I_{2}\right\}
$$

Then inequality (32) implies that we can find $k(u)>1$ such that

$$
\begin{cases}I_{2}(t u)>a & \text { if } t \in[0, k(u)), \\ I_{2}(t u)=a & \text { if } t=k(u), \\ I_{2}(t u)<a & \text { if } t>k(u) .\end{cases}
$$

Moreover, the implicit function theorem implies that $k \in C\left(\partial B_{1,+}^{c},[1,+\infty)\right)$.

By the choice of $a$, we have

$$
I_{2}^{a}=\left\{t u: u \in \partial B_{1,+}^{c}, t \geq k(u)\right\}
$$

We define the set $E_{+}=\left\{t u: u \in \partial B_{1,+}^{c}, t \geq 1\right\}$. The map $\hat{h}_{+}:[0,1] \times E_{+} \rightarrow E_{+}$defined by

$$
\hat{h}_{+}(s, t u)=\left\{\begin{array}{ll}
(1-s) t u+s k(u) u & \text { if } 1 \leq t<k(u), \\
t u & \text { if } t \geq k(u),
\end{array} \quad s \in[0,1]\right.
$$

is a continuous deformation of $E_{+}, \hat{h}_{+}\left(1, E_{+}\right) \subset I_{2}^{a}$ and $\left.\hat{h}_{+}(s, \cdot)\right|_{I_{2}^{a}}=\left.i d\right|_{I_{2}^{a}}$ for all $s \in[0,1]$ (see equations (34) and (35)). Therefore, $I_{2}^{a}$ is a strong deformation retract of $E_{+}$. Hence we have

$$
H_{k}\left(C_{0}^{3}(\bar{\Omega}), I_{2}^{a}\right)=H_{k}\left(C_{0}^{3}(\bar{\Omega}), E_{+}\right)=H_{k}\left(C_{0}^{3}(\bar{\Omega}), \partial B_{1,+}^{c}\right) \quad \text { for all } k \geq 0 \text {. }
$$


Recalling that in the first part of the proof, we established that $\partial B_{1,+}^{c}$ is contractible. This yields

$$
H_{k}\left(C_{0}^{3}(\bar{\Omega}), \partial B_{1,+}^{c}\right)=0 \quad \text { for all integers } k \geq 0 .
$$

Combining with equation (36) leads to equation (22), which completes the proof.

Proposition 3.4 If the assumptions of Theorem 2.1 hold, then

$$
C_{d}(I, 0) \neq 0,
$$

where $d=\operatorname{dim} V(V$ being defined in Lemma 2.3).

Proof By condition $\left(\mathrm{H}_{5}\right)$, given $\epsilon>0$, we can find $\delta_{*}>0$ such that

$$
\frac{1}{2}\left(\vartheta_{1}(x)-\epsilon\right) t^{2} \leq F(x, t) \quad \text { for all } x \in \Omega \text { and all }|t| \leq \delta_{*} .
$$

Since $V$ is finite dimensional, all norms are equivalent. Thus we can find $\rho>0$ small such that

$$
\|u\| \leq \rho \quad \Leftrightarrow \quad\|u\|_{\infty} \leq \delta_{*}
$$

for all $u \in V$. Taking inequalities (37) and (38) into account, for all $u \in V$ with $\|u\| \leq \rho$ we have

$$
I(u) \leq \frac{1}{2} \int_{\Omega}\left(\lambda_{k}-\vartheta_{1}(x)\right) u^{2} d x+\frac{\epsilon}{2}|u|_{2}^{2} .
$$

Similar to the proof of Lemma 2.3, there exists $C>0$ such that

$$
I(u) \leq(-C+\epsilon)\|u\|^{2} \leq 0
$$

for all $u \in V$ and $\|u\| \leq \rho$.

On the other hand, for given $\epsilon>0$, it follows from $\left(\mathrm{H}_{2}\right)$ and $\left(\mathrm{H}_{5}\right)$ that

$$
F(x, t) \leq \frac{\vartheta_{2}(x)+\epsilon}{2} t^{2}+C_{\epsilon}|t|^{r}
$$

for all $x \in \Omega$ and $t \in R$. By (41) and Lemma 2.3, we have

$$
I(u) \geq C_{4}\|u\|^{2}-C_{5}\|u\|^{r}
$$

for all $u \in W$. From inequality (42), we infer that for $\rho$ small enough we have

$$
I(u)>0 \quad \text { for all } u \in W \text { with } 0<\|u\| \leq \rho .
$$

From inequalities (40) and (43), we know that $I$ has a local linking at 0 . Then invoking Proposition 2.3 of Bartsch and Li [17], we obtain $C_{d}(I, 0) \neq 0$. 


\section{Proof of the main result}

Proof of Theorem 2.1 We consider the following problem:

$$
\left\{\begin{array}{l}
\triangle^{2} u+c \Delta u=f_{-}(x, u), \quad x \in \Omega \\
\left.u\right|_{\partial \Omega}=\left.\Delta u\right|_{\partial \Omega}=0
\end{array}\right.
$$

where

$$
f_{-}(x, t)= \begin{cases}f(x, t), & t<0 \\ 0, & t \geq 0\end{cases}
$$

Define a functional $I_{-}: E=H^{2}(\Omega) \cap H_{0}^{1}(\Omega) \rightarrow \mathbb{R}$ by

$$
I_{-}(u)=\frac{1}{2} \int_{\Omega}\left(|\Delta u|^{2}-c|\nabla u|^{2}\right) d x-\int_{\Omega} F_{-}(x, u) d x,
$$

where $F_{-}(x, t)=\int_{0}^{t} f_{-}(x, s) d s$, then $I_{-} \in C^{2}(E, \mathbb{R})$. Obviously, by conditions $\left(\mathrm{H}_{1}\right)$ and $\left(\mathrm{H}_{3}\right)$, we know that $I_{-}$is coercive and boundedness from below. Thus we can find $v_{0} \in E$ such that

$$
I_{-}\left(v_{0}\right)=\inf I_{-}=: m_{-} .
$$

Next, we claim that $v_{0} \neq 0$. By condition $\left(\mathrm{H}_{5}\right)$, given $\epsilon \in\left(0, \lambda_{k}-\lambda_{1}\right)$, there exists $\delta>0$ such that

$$
\frac{1}{2}\left(\vartheta_{1}(x)-\epsilon\right) t^{2} \leq F_{-}(x, t) \quad \text { for all } x \in \Omega, t \in[-\delta, 0]
$$

For $s$ small enough, it follows from inequality (45) that

$$
\begin{aligned}
I_{-}\left(-s \phi_{1}\right) & =\frac{s^{2}}{2} \lambda_{1}-\int_{\Omega} F_{-}\left(x,-s \phi_{1}\right) d x \\
& \leq \frac{s^{2}}{2}\left(\lambda_{1}-\lambda_{k}+\epsilon\right)<0,
\end{aligned}
$$

and thus, by equation $(44), I_{-}\left(v_{0}\right) \leq I_{-}\left(-s \phi_{1}\right)<0$, so $v_{0} \neq 0$. From condition $\left(\mathrm{H}_{1}\right)$ and strong maximum principle, we have $v_{0}<0$ and

$$
C_{k}\left(I_{-}, v_{0}\right)=\delta_{k, 0} \mathbb{Z} \quad \text { for all integers } k \geq 0 \text {. }
$$

Since $v_{0}$ is an interior point of $-C_{+}$, from Proposition 3.1, we know

$$
C_{k}\left(I, v_{0}\right)=C_{k}\left(I_{-}, v_{0}\right)=\delta_{k, 0} \mathbb{Z} \quad \text { for all integers } k \geq 0 \text {. }
$$

Let $\theta \in \mathbb{R}, \epsilon>0$ be such that $\theta<m_{-}=I\left(v_{0}\right)<-\epsilon$. We consider the sublevel sets

$$
I^{\theta} \subset I^{-\epsilon} \subset I^{\epsilon} \subset E .
$$

Suppose that 0 and $v_{0}$ are the only critical points of $I$. Otherwise, we have a second nontrivial smooth solution and so we are done. By Proposition 3.3, we have

$$
H_{k}\left(E, I^{\theta}\right)=C_{k}(I, \infty)=0 \quad \text { for all integers } k \geq 0 \text {. }
$$


We know that $I$ satisfies the (PS) condition (see Lemma 2.2). Hence choosing $\epsilon>0$ small enough, we have

$$
H_{d}\left(I^{\epsilon}, I^{-\epsilon}\right)=C_{d}(I, 0) \neq 0
$$

(see Proposition 3.4). Because of equations (47) and (48), using Proposition 3.2, we obtain

$$
H_{d+1}\left(E, I^{\epsilon}\right) \neq 0 \quad \text { or } \quad H_{d-1}\left(I^{-\epsilon}, I^{\theta}\right) \neq 0 .
$$

If $H_{d+1}\left(E, I^{\epsilon}\right) \neq 0$, then there is a critical point $v^{*} \in E$ of $I$ such that

$$
I\left(v^{*}\right)>\epsilon>0 \quad \text { and } \quad v^{*} \neq 0, v_{0} .
$$

If $H_{d-1}\left(I^{-\epsilon}, I^{\theta}\right) \neq 0$, then there is a critical point $v^{*} \in E$ of $I$ such that

$$
C_{d-1}\left(I, v^{*}\right) \neq 0 .
$$

Since $d \geq 2$, from equations (46) and (49), we see that $v^{*} \neq v_{0}$. It is obvious that $v^{*} \neq 0$. Therefore $v_{0}$ and $v^{*}$ are two solutions of problem (1).

\section{Competing interests}

The authors declare that they have no competing interests.

\section{Authors' contributions}

The authors read and approved the final manuscript.

\section{Acknowledgements}

The authors would like to thank the referees for valuable comments and suggestions in improving this article. This study was supported by the National NSF (Grant No. 11101319) of China and Planned Projects for Postdoctoral Research Funds of Jiangsu Province (Grant No. 1301038C)

Received: 14 August 2013 Accepted: 16 December 2013 Published: 10 Jan 2014

\section{References}

1. Các, NP: On nontrivial solutions of a Dirichlet problem whose jumping nonlinearity crosses a multiple eigenvalue. J. Differ. Equ. 80, 379-404 (1989)

2. Dancer, EN, Zhang, Z: Fučík spectrum, sign-changing, and multiple solutions for semilinear elliptic boundary value problems with resonance at infinity. J. Math. Anal. Appl. 250, 449-464 (2000)

3. Magalhães, CA: Multiplicity results for a semilinear elliptic problem with crossing of multiple eigenvalues. Differ. Integral Equ. 4, 129-136 (1991)

4. de Paiva, FO: Multiple solutions for a class of quasilinear problems. Discrete Contin. Dyn. Syst. 15, 669-680 (2006)

5. Schechter, M: The Fučík spectrum. Indiana Univ. Math. J. 43, 1139-1157 (1994)

6. Arcoya, D, Villegas, S: Nontrivial solutions for a Neumann problem with a nonlinear term asymptotically linear at $-\infty$ and superlinear at $+\infty$. Math. Z. 219, 499-513 (1995)

7. Perera, K: Existence and multiplicity results for a Sturm-Liouville equation asymptotically linear at $-\infty$ and superlinear at $+\infty$. Nonlinear Anal. 39, 669-684 (2000)

8. Motreanu, D, Motreanu, W, Papageorgiou, NS: Multiple solutions for Dirichlet problems which are superlinear at $+\infty$ and (sub-) linear at $-\infty$. Commun. Pure Appl. Anal. 13, 341-358 (2009)

9. Ziemer, WP: Weakly Differentiable Functions. Grad. Texts in Math., vol. 120. Springer, Berlin (1989)

10. Liu, Y, Wang, ZP: Biharmonic equations with asymptotically linear nonlinearities. Acta Math. Sci. 27, $549-560$ (2007)

11. Pei, RC: Multiple solutions for biharmonic equations with asymptotically linear nonlinearities. Bound. Value Probl. 2010, Article ID 241518 (2010)

12. Lazer, AC, Mckenna, PJ: Large amplitude periodic oscillation in suspension bridges: some new connections with nonlinear analysis. SIAM Rev. 32, 537-578 (1990)

13. Micheletti, AM, Pistoia, A: Multiplicity solutions for a fourth order semilinear elliptic problems. Nonlinear Anal. TMA 31, 895-908 (1998)

14. Chipot, M: Variational Inequalities and Flow in Porous Media. Springer, New York (1984)

15. Zhang, JH, Li, SJ: Multiple nontrivial solutions for some fourth-order semilinear elliptic problems. Nonlinear Anal. TMA 60, 221-230 (2005) 
16. Chang, KC: Infinite Dimensional Morse Theory and Multiple Solutions Problems. Birkhäuser, Boston (1993)

17. Bartsch, T, Li, SJ: Critical point theory for asymptotically quadratic functionals and applications to problems with resonance. Nonlinear Anal. TMA 28, 419-441 (1997)

18. Qian, AX: Multiple solutions for a fourth-order asymptotically linear elliptic problem. Acta Math. Sin. 22, 1121-1126 (2006)

19. Perera, K: Critical groups of critical points produced by local linking with applications. Abstr. Appl. Anal. 3, 437-446 (1998)

20. Palais, RS: Homotopy theory of infinite dimensional manifolds. Topology 5, 1-16 (1966)

10.1186/1687-2770-2014-12

Cite this article as: Pei and Zhang: Multiple solutions for a fourth-order nonlinear elliptic problem which is superlinear at $+\infty$ and linear at $-\infty$. Boundary Value Problems 2014, 2014:12

Submit your manuscript to a SpringerOpen ${ }^{\circ}$ journal and benefit from:

- Convenient online submission

- Rigorous peer review

- Immediate publication on acceptance

- Open access: articles freely available online

- High visibility within the field

- Retaining the copyright to your article 